\title{
Numerical simulations of kink instability in line-tied coronal Loops
}

\author{
C. L. Gerrard ${ }^{1}$, T. D. Arber ${ }^{2}$, A. W. Hood ${ }^{1}$, and R. A. M. Van der Linden ${ }^{3}$ \\ 1 School of Mathematics and Statistics, University of St Andrews, North Haugh, St Andrews, Fife KY16 9SS, \\ Scotland \\ 2 Space and Astrophysics Group,Physics Department, University of Warwick, Coventry, CV4 7AL, UK \\ 3 Koninklijke Sterrenwacht van Belgie, Ringlaan 3, 1180 Ukkel, Belgium
}

Received 13 December 2000 / Accepted 9 May 2001

\begin{abstract}
The results from numerical simulations carried out using a new shock-capturing, Lagrangian-remap, 3D MHD code, Lare3d are presented. We study the evolution of the $m=1$ kink mode instability in a photospherically line-tied coronal loop that has no net axial current. During the non-linear evolution of the kink instability, large current concentrations develop in the neighbourhood of the infinite length mode rational surface. We investigate whether this strong current saturates at a finite value or whether scaling indicates current sheet formation. In particular, we consider the effect of the shear, defined by $r \phi^{\prime} / \phi$ where $\phi=L B_{\theta} / r B_{z}$ is the fieldline twist of the loop, on the current concentration. We also include a non-uniform resistivity in the simulations and observe the amount of free magnetic energy released by magnetic reconnection.
\end{abstract}

Key words. MHD - Sun

\section{Introduction}

Solar flares are a clear manifestation of the dramatic conversion of magnetic energy into heat and motion. Large two-ribbon flares involve the complete destruction of a magnetic structure and are frequently associated with prominence eruptions and coronal mass ejections. Smaller compact loop flares appear as a brightening in an individual loop but the loop structure is not destroyed and the energy released is substantially smaller. Both of these types of flares occur extremely rapidly, on a timescale comparable to the coronal Alfvén timescale, and involve the release of magnetic energy. This paper is concerned with the non-linear magnetic instabilities which may be responsible for compact loop flares.

The Alfvén timescale, $\tau_{\mathrm{A}}$, for a loop of length $L=5 \times$ $10^{7} \mathrm{~m}$, magnetic field strength of 100 Gauss and number density $5 \times 10^{14} \mathrm{~m}^{-3}$ is of the order of a few seconds. This is comparable to the rise time at the start of a flare, suggesting that the initial instability is due to an Alfvénic process. An obvious candidate for generating such fast processes is an an ideal MHD instability. However, the problem here is that the magnetic topology is conserved in an ideal MHD and the amount of magnetic energy that can be released as kinetic energy is quite small. Resistivity must be

Send offprint requests to: C. L. Gerrard,

e-mail: cath@mcs.st-and.ac.uk included so that field line topologies with lower magnetic energy can be reached by reconnection, releasing kinetic energy and, through ohmic dissipation, heat.

Magnetic reconnection can occur in several ways. For example, it may occur through the non-linear development of a resistive instability, such as the tearing mode. However the timescale for this is of the order $\sqrt{\tau_{\mathrm{A}} \tau_{\mathrm{d}}}$, where $\tau_{\mathrm{A}}$ is the Alfvén timescale defined above and $\tau_{\mathrm{d}}=L^{2} / \eta$ is the magnetic diffusion timescale. For typical coronal conditions the tearing mode timescale is the order of a day and, hence, is too slow to be responsible for a compact loop flare. Alternatively, magnetic reconnection can be driven and, indeed, may be driven at the Alfvén speed making it a viable mechanism for magnetic energy release. Reconnection only occurs when the gradients in the magnetic field are sufficiently large. The required magnitude of these gradients depends on the Lundquist number, defined as $S=\tau_{\mathrm{d}} / \tau_{\mathrm{A}}$, and must be larger if the value of the Lundquist number is larger. In this paper we refer to the Lundquist number, rather than the more commonly used magnetic Reynolds number, because we use the Alfvén speed as the reference speed rather than a typical flow speed. The key problem, in this case, is to explain how reconnection can be driven on an Alfvénic timescale. A possible mechanism is the build-up of large gradients in the current in a narrow region as a consequence of the non-linear development of an ideal MHD instability. Thus, 
reconnection can occur and the magnetic field can be carried, by the instability, into the reconnection region on a timescale related to the growth time of an ideal MHD instability, namely the order of the Alfvénic timescale. For further details of considerations about reconnection see Priest \& Forbes (2000) and references therein.

So magnetic reconnection may be driven by a build-up of current due to an ideal MHD instability. The idea is attractive but it does depend on the non-linear development of the instability producing a region with large current. If the instability saturates and the maximum current reaches a finite value, then the Lundquist number must be smaller than a particular, critical value for reconnection to occur. The value of the coronal resistivity, and hence the Lundquist number, is unknown making it difficult to estimate this critical value. However, if the ideal MHD instability produces a current sheet, an infinitely thin region of infinite current density in the strict mathematical sense through which the magnetic field changes direction, then, regardless of the value of the coronal resistivity, reconnection will always occur and magnetic energy will be converted into heat and motion. Hence, numerical simulations based on a larger value of the resistivity will produce qualitatively correct results. Thus, it is important to understand the conditions under which the non-linear stage of an ideal MHD instability produces a current sheet.

At this point it may be useful to explain the difference between a current sheet and a current concentration. A current concentration is a large build up of current but the current will saturate at some value whereas for a current sheet the current is infinite and therefore there is no saturation. Numerically a current sheet can be recognised through the fact that the maximum value of the current will increase with higher grid resolution. Therefore to check for current sheet formation, we carry out simulations on different grid resolutions and see how the maximum current scales. If it does not continually increase with higher resolution, flattening off at some value then we have saturation of the current and a current concentration has formed rather than a current sheet.

There is compelling theoretical evidence that once the electron fluid slow speed, $v_{\mathrm{e}}$, exceeds the phase velocity of the ion-acoustic mode, $c_{\mathrm{ia}}$, that ion-acoustic turbulence would have a profound effect on current sheet development. Indeed Bychenkov et al. (1988) have shown that under a wide range of conditions the effective anomalous resistivity is a function of $\boldsymbol{E}$ (or $\boldsymbol{j}$ ) and adjusts to keep $v_{\mathrm{e}} \leq c_{\mathrm{ia}}$. Thus an effective formula for anomalous resistivity would be,

$\eta=\eta_{0} \operatorname{MAx}\left(0, \frac{\left|v_{\mathrm{e}}\right|}{c_{\mathrm{ia}}}-1\right)$.

In this paper we assume that $\left|v_{\mathrm{e}}\right| \sim \frac{|j|}{n e}$ and take,

$\eta=\eta_{0} \operatorname{MAX}\left(0, \frac{|j|}{j_{\text {crit }}}-1\right)$,

where $j_{\text {crit }}=n e c_{\mathrm{ia}}$.
This theory would suggest that the largest current density which would evolve in an unstable loop would have $j_{\max } \sim$ $n e c_{\mathrm{ia}}$. If we take,

$j=\frac{B_{0}}{\mu_{0} L}$

then to reach this $j_{\text {crit }}$ we would require a scale length collapse to $L_{\min }$ where,

$L_{\min }=\frac{B_{0}}{\mu_{0} n e c_{\mathrm{ia}}}$.

For $B_{0}=100 \mathrm{G}, n=5 \times 10^{14} \mathrm{~m}^{-3}$ and $c_{\mathrm{ia}}=1.35 \times$ $10^{5} \mathrm{~ms}^{-1}$ this gives $L=765 \mathrm{~m}$. Taking the loop radius to be $\sim 1 \mathrm{Mm}$ this gives a difference in scale lengths of around 1300. Thus to fully resolve current densities up to $j_{\text {crit }}$ would require grids of around $5000^{3}$. This estimate is based on a uniform grid. Allowing for stretched grids this can be reduced but only to around $3000^{3}$. This is because the higher resolution is needed around the rational surface but Lare3d uses a Cartesian grid so this amounts to having a fine, but uniform, grid over the central region and then stretching to larger spacing in the potential field outer region. The central region must still be capable of resolving the 1300 times difference in scale lengths and the boundary must be remote from the central column. This problem would be ideally suited to an adaptive mesh approach but Lare3d is currently fixed grid. Here we are limited to $251^{3}$ grids and so cannot reach the fully resolved limit. Our measure of current concentration formation is taken throughout to be $j_{\max }$, the infinite norm supremum current density. In the continuous limit this is itself undefined. However, for the finite resolutions considered here, this is just the maximum grid based current density and is a sensible, and finite, measure.

As in previous work, the equilibrium coronal loop is modelled as a cylindrical structure with axial, $B_{z}$, and azimuthal, $B_{\theta}$, magnetic field components that are just functions of the radial co-ordinate. Linear stability theory has shown that a current sheet is likely to form at a mode rational surface if the loop is of infinite length. For perturbations of the form

$f(\boldsymbol{r}, t)=f(r) \mathrm{e}^{i(m \theta+k z-\omega t)}$,

a mode rational surface is the radius, for given values of the wavenumbers $m$ and $k$, at which

$\boldsymbol{k} \cdot \boldsymbol{B}=\frac{m}{r} B_{\theta}+k B_{z}=0$.

However, coronal loops do not have an infinite length and the ends of the magnetic fields, the footpoints, are located in the dense solar photosphere. This means that the coronal magnetic field lines are inertially line-tied to the photosphere and the simplest way of simulating the dense photosphere is to assume that all components of the plasma velocity vanish at the footpoints. Discussions of the relevant photospheric boundary conditions can be found in Hood (1986); Velli et al. (1990); Van der Linden et al. (1994). 
One immediate consequence of photospheric line-tying is that mode rational surfaces do not exist mathematically (Velli et al. 1990). However, the linear instabilities still exhibit regions where the current gradient does indeed buildup. The non-linear behaviour is not really known and it is important to determine whether current sheet formation is a natural consequence of an ideal MHD instability when photospheric line-tying is included.

The three-dimensional evolution of the kink instability in coronal loops has recently been investigated by various authors. Some of these (Baty \& Heyvaerts 1996; Baty 1997a,b; Baty et al. 1998) found that a non-singular current concentration formed during the non-linear evolution of the instability. In contrast, other authors (Bazdenkov \& Sato 1998; Lionello et al. 1998; Arber et al. 1999; Baty 2000a,b) have observed indications of current sheet formation rather than the formation of a non-singular current concentration.

In this paper we again investigate the question of current sheet formation. In particular we consider the suggestion of Baty (2000a) that saturation of the current may be difficult to observe for loops of large shear and that this may explain the apparently contradictory results on current sheet formation during the non-linear evolution of the kink instability. We, therefore, consider two initial equilibria one of which has shear of 6.32 whilst the second is defined such that we can vary the shear. We present results for the second equilibrium with shear between 1.67 and 9.42. We carry out the numerical simulations using a new 3D MHD code, Lare3d (Arber et al. 1999) which is described in Sect. 2. The equilibria are defined in Sect. 3 and the results for the non-linear and resistive evolution are presented in Sect. 4. Finally in Sect. 5 we discuss how the shear affects the non-linear evolution. In particular, we concentrate on whether we observe saturation of the currents or scaling indicative of current sheet formation and present results for the resistive evolution of the instability.

\section{Numerical details}

The non-linear evolution of the loop is modelled by the MHD equations,

$$
\frac{\partial \rho}{\partial t}=-\nabla \cdot(\rho \boldsymbol{v})
$$

$\frac{\partial}{\partial t}(\rho \boldsymbol{v})=-\nabla \cdot(\rho \boldsymbol{v} \boldsymbol{v})+\frac{1}{\mu_{0}}(\nabla \times \boldsymbol{B}) \times \boldsymbol{B}-\nabla P$,

$\frac{\partial \boldsymbol{B}}{\partial t}=\nabla \times(\boldsymbol{v} \times \boldsymbol{B})-\nabla \times\left(\eta \frac{\nabla \times \boldsymbol{B}}{\mu_{0}}\right)$,

$\frac{\partial}{\partial t}(\rho \epsilon)=-\nabla \cdot(\rho \epsilon \boldsymbol{v})-P \nabla \cdot \boldsymbol{v}+\eta j^{2}$,

with specific energy density,

$\epsilon=\frac{P}{(\gamma-1) \rho}$.
$\boldsymbol{B}$ is the magnetic field, $\boldsymbol{j}=(\nabla \times \boldsymbol{B}) / \mu_{0}$ is the current density, $\boldsymbol{v}$ is the velocity, $P$ is the thermal pressure, $\epsilon$ is the specific energy density $(\gamma=5 / 3), \rho$ is the mass density, $\eta$ is the resistivity, and $\mu_{0}=4 \pi \times 10^{-7}$ is the magnetic permittivity. We ignore the effects of thermal conduction, radiation and heating, apart from ohmic heating. Also since the scale height in the corona is relatively large (approximately $100 \mathrm{Mm}$ ) compared to the height of the loops $(10-50 \mathrm{Mm})$ we neglect the effect of gravity.

The equations are made dimensionless by setting, $\boldsymbol{r} \longrightarrow r^{*} \tilde{\boldsymbol{r}}, \quad \boldsymbol{B} \longrightarrow B^{*} \tilde{\boldsymbol{B}}, \quad \boldsymbol{v} \longrightarrow v_{A} \tilde{\boldsymbol{v}}$ $P \longrightarrow P^{*} \tilde{P}, \quad t \longrightarrow t^{*} \tilde{t}, \quad \rho \longrightarrow \rho^{*} \tilde{\rho}, \quad \eta \longrightarrow \eta^{*} \tilde{\eta}$, where a tilde denotes a dimensionless variable. $v_{\mathrm{A}}$ is the Alfvén speed given by $v_{\mathrm{A}}=B^{*} / \sqrt{\mu \rho^{*}}, t^{*}=r^{*} / v_{\mathrm{A}}$ is the Alfvén transit time, $P^{*}=B^{* 2} / \mu$. Often the normalisation adopted in MHD studies is to take $\eta^{*}=\mu_{0} r^{*} v_{\mathrm{A}} / S$ so that for uniform resistivity we can write Eq. (8) as,

$\frac{\partial \boldsymbol{B}}{\partial t}=\nabla \times(\boldsymbol{v} \times \boldsymbol{B})+\frac{1}{S} \nabla^{2} \boldsymbol{B}$,

and then specify $S$. However, as already discussed we do not use uniform resistivity and consequently we have kept a normalised $\eta$ explicitly in the equations by taking $\eta^{*}=\mu_{0} r^{*} v_{\mathrm{A}}$. This is then chosen to prevent $|j|$ from greatly exceeding $j_{\text {crit }}$, for reasons already discussed earlier and in Arber et al. (1999). Thus we obtain the dimensionless equations, removing the tildes from the dimensionless quantities,

$\frac{\partial \rho}{\partial t}=\nabla \cdot(\rho \boldsymbol{v})$,

$\frac{\partial}{\partial t}(\rho \boldsymbol{v})=-\nabla \cdot(\rho \boldsymbol{v} \boldsymbol{v})+(\nabla \times \boldsymbol{B}) \times \boldsymbol{B}-\nabla P$,

$\frac{\partial \boldsymbol{B}}{\partial t}=\nabla \times(\boldsymbol{v} \times \boldsymbol{B})-\nabla \times(\eta \nabla \times \boldsymbol{B})$,

$\frac{\partial}{\partial t}(\rho \epsilon)=-\nabla \cdot(\rho \epsilon \boldsymbol{v})-P \nabla \cdot \boldsymbol{v}+\eta j^{2}$.

We consider both the ideal evolution $(\eta=0)$ and the resistive evolution of the instability. The choice of the form for the resistivity follows Arber et al. (1999). We take, in dimensionless variables,

$\eta=\eta_{0} \operatorname{MAx}\left(0, \frac{|j|}{j_{\text {crit }}}-1\right)$,

such that the resistivity will be switched on only once the current has exceeded some critical value.

The simulations are carried out using a 3D MHD, Lagrangian remap, shock capturing code (Lare3d). The Lagrangian step is fully $3 \mathrm{D}$, uses the predictor-corrector method and artificial viscosity. The remap step uses Van Leer gradient limiters (Van Leer 1997), applied to the density, specific energy density, velocities and magnetic fluxes, to ensure that it is monotonicity preserving. Furthermore, Lare3d uses Evans and Hawley constrained transport (Evans \& Hawley 1988) to guarantee that if $\nabla . B$ is initially zero it is maintained at zero to 
machine precision throughout the evolution. The numerical grid is staggered so that the density, pressure and specific energy density are defined at the cell centres; the velocities at the vertices; the magnetic field components at the cell faces and the current components along the edges of the numerical cell. $|j|=\left(j_{x}^{2}+j_{y}^{2}+j_{z}^{2}\right)^{\frac{1}{2}}$ and the resistivity are defined at the same vertices as the velocities. The staggered grid reduces the amount of averaging required in some of the calculations, thus reducing the associated error, and removes chequerboard biasing. Further details of the code are given in Arber et al. (2001). This code has many similarities with the ZEUS code (Stone \& Norman 1992a,b). The major difference is that by using a second order Lagrangian step to treat all of the physics there is no need to adopt MOC techniques to upwind in the Alfvén waves. Indeed, there is no upwinding at all as the Lagrangian step does not include advection terms. The remap step is the only stage in which Van Leer limiters are needed and this is purely geometrical, i.e. upwinding is not an issue. The velocity components are also defined on cell vertices, not face centred as in ZEUS, to reduce checkerboard biasing.

As in previous simulations, the coronal loop is modelled as an initially straight cylinder. This can be justified as coronal loops generally have aspect ratios (ratio of length to width) of order 10 and it also allows for comparison with previous work. The loop has length $L_{z}$ with line-tying boundary conditions imposed at $z=-L_{z} / 2$ and $z=L_{z} / 2$. The code is written in Cartesian co-ordinates with the numerical box stretching from $-L_{x} / 2$ to $L_{x} / 2$ and $-L_{y} / 2$ to $L_{y} / 2$. While not the appropriate geometry for the linear instabilities the use of Cartesian geometry does remove the problems associated with the singular nature of the axis at $r=0$. Cylindrical co-ordinates would be preferable for the equilibrium but the $m=1$ instability corresponds to a lateral displacement of the axis and thus the $1 / r$ dependence would be a problem. Also, we had no prior reason to think that the fully non-linear developed stage would preserve any cylindrical symmetry. The use of Cartesian co-ordinates does have limitations but has been shown to reproduce the cylindrically symmetric eigenvalues (Arber et al. 1999) and to accurately find $m=0$ and $m=1$ growth rates. The values of $L_{x}$ and $L_{y}$ are chosen such that the boundary conditions imposed in the $x$ and $y$ directions have no effect on the evolution of the loop, which remains localised within a smaller region.

We solve the linear equations to establish the critical lengths for the equilibria and the form of the eigenmodes. Solving the Hain-Lust equations (Goedbloed 1983), gives the maximum growth rate and the corresponding value of the axial wavenumber, $k$. From the linearised MHD equations for line-tied loops of finite length, we find the growth rates for finite lengths of the loop, the length for marginal stability and the form of the eigenfunctions. From these results we can choose the length of the loop, $L_{z}$, such that it is unstable and we can use an initial velocity perturbation based on the eigenmodes to speed up the development of the instability.
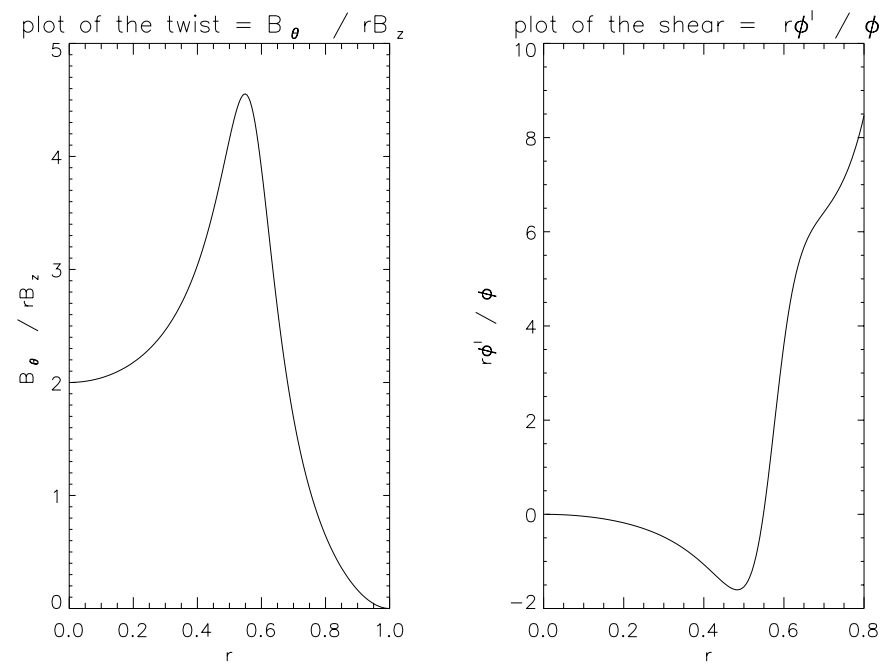

Fig. 1. The twist and shear of Equilibrium 1.

\section{Equilibria}

In this paper we consider two equilibria. The first has a shear of 6.32 at the mode rational surface, where the shear is defined as

shear $=\frac{r \Phi^{\prime}}{\Phi}$

the twist is defined by,

$\Phi=\frac{L_{z} B_{\theta}}{r B_{z}}$

The second equilibrium was chosen so that we could vary the shear and thus investigate the effect of shear on the formation of current sheets.

\subsection{Equilibrium 1}

Equilibrium 1, is defined by specifying $B_{\theta}$,

$B_{\theta}=\left\{\begin{array}{cc}r\left(1-r^{2}\right)^{2} & r<1.0 \\ 0.0 & r \geq 1.0\end{array}\right.$

This gives $B_{\theta}=0.0$ at $r=1.0$ and $j_{z}=0.0$ at $r=1.0$ - there is no net axial current in the loop. The axial field component is obtained from,

$B_{z}^{2}=B_{0}^{2}-B_{\theta}^{2}-\int_{0}^{r} 2 \frac{B_{\theta}^{2}}{u} \mathrm{~d} u$

as,

$B_{z}=\left\{\begin{array}{cc}\sqrt{B_{0}^{2}-\frac{1}{5}-r^{2}\left(1-r^{2}\right)^{4}+\frac{1}{5}\left(1-r^{2}\right)^{5}} & r<1.0, \\ \sqrt{B_{0}^{2}-\frac{1}{5}} & r \geq 1.0 .\end{array}\right.$

$B_{z}^{2}$ must remain positive hence we choose $B_{0}=0.5$, and run the equilibrium for a uniform density of 1.0. We consider four forms for the pressure: pressure, $P=0(\beta=0)$ and pressure given by a plasma $\beta$ of $10^{-2}, 10^{-3}$ and $10^{-4}$. This initial equilibrium configuration gives the twist and shear profiles shown in Fig. 1. 
Since the equilibrium current is confined within a radius of 1 we take $L_{x}=L_{y}=5$ and we stretch the grid in the $x$ and $y$ directions such that $50 \%$ of the points lie within $r=1.1$. The results for the linear evolution provide the critical length of the loop and the form of a suitable velocity perturbation to start the non-linear simulations. Thus, $L_{z}=3 \pi$ since the critical half-length of the loop is approximately 2.2. This gives a length of loop which exceeds the critical length guaranteeing that the loop is unstable to the $m=1$ kink mode and taking this larger value for the length speeds up the evolution of the instability.

\subsection{Equilibrium 2}

The second equilibrium configuration (Equilibrium 2) is chosen so that the value of the shear may be chosen as either small $(<6)$ or large $(>6)$. This equilibrium is defined by specifying the twist,

$\Phi=\left\{\begin{array}{cc}\Phi_{0} & r<a, \\ \frac{\Phi_{0}}{2}\left(1+\cos \pi\left(\frac{r-a}{b-a}\right)\right) & a<r<b, \\ 0.0 & r>b,\end{array}\right.$

where $a, b$ and $\Phi_{0}$ are variables. The magnetic field components can then be calculated from,

$\log B_{z}=-\frac{1}{2} \log \left(1+r^{2} \Phi^{2}\right)-\int_{0}^{r} \frac{r \Phi^{2}}{1+r^{2} \Phi^{2}} \mathrm{~d} r$,

and,

$B_{\theta}=r \Phi B_{z}$.

The integral in Eq. (23) is evaluated numerically. As above, the density is assumed uniform and the plasma pressure is taken as zero. The values of $a, b$, and $\Phi_{0}$ are varied to adjust the value of the shear at the mode rational surface (whose position also varies with $a, b$ and $\left.\Phi_{0}\right)$. Figure 2 shows the twist and shear profiles for the configuration with $\Phi_{0}=1.0, a=0.5$ and $b=2.0$. Unlike Equilibrium 1, the twist is a monotonically decreasing function of radius. For this equilibrium we take $L_{x}=L_{y}=5.0$ and $L_{z}=10 \pi$ and stretch the grid as for Equilibrium 1. Again we use an initial velocity perturbation based on the linear mode to speed up the development of the instability.

\section{Results}

\subsection{Equilibrium 1}

\subsubsection{Non-linear evolution}

The non-linear evolution of the instability for Equilibrium 1 is followed using two non-linear MHD codes. The first is Lare3d. The second is an ideal MHD Lagrangian code described in Arber et al. (1999).

We run Lare3d for 40 Alfvén times using Equilibrium 1 as the initial equilibrium on an $81^{3}$, a $121^{3}$, a $161^{3}$ and a
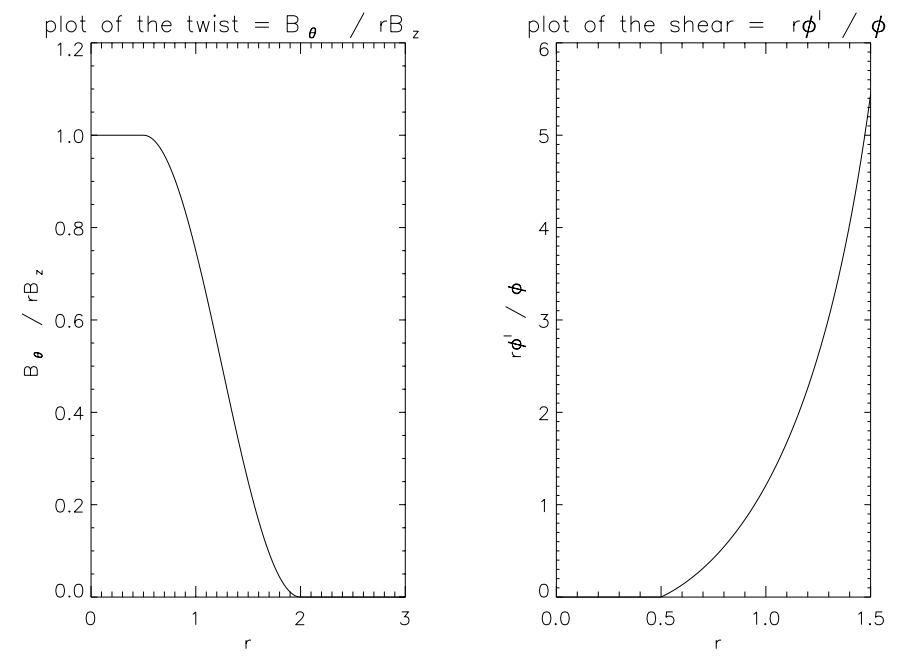

Fig. 2. The twist and shear profiles for Equilibrium 2 with $\Phi_{0}=1.0, a=0.5$ and $b=2.0$.

$251^{3}$ grid. This allows us to investigate the scaling of the current with higher resolution. For the $\beta=0$ case we find a growth rate of 0.11 whilst for the $\beta=10^{-2}$ case we find a reduced growth rate of 0.09 . The $\beta=10^{-2}$ case evolves more slowly and hence has smaller currents but otherwise there seems to be no major difference between the two cases. For the $\beta=0$ case we find that the current starts to build up after 5 Alfvén times at $r=0.70$ and is shifted outwards to $r=0.75$ as shown in Fig. 3. This shows that if we have current sheet formation then it occurs near the mode rational surface $(r=0.69)$ as would be expected. During the non-linear evolution of the kink instability the whole loop is moved by the instability and since the location of the mode rational surface is shifted the position of the current is also shifted.

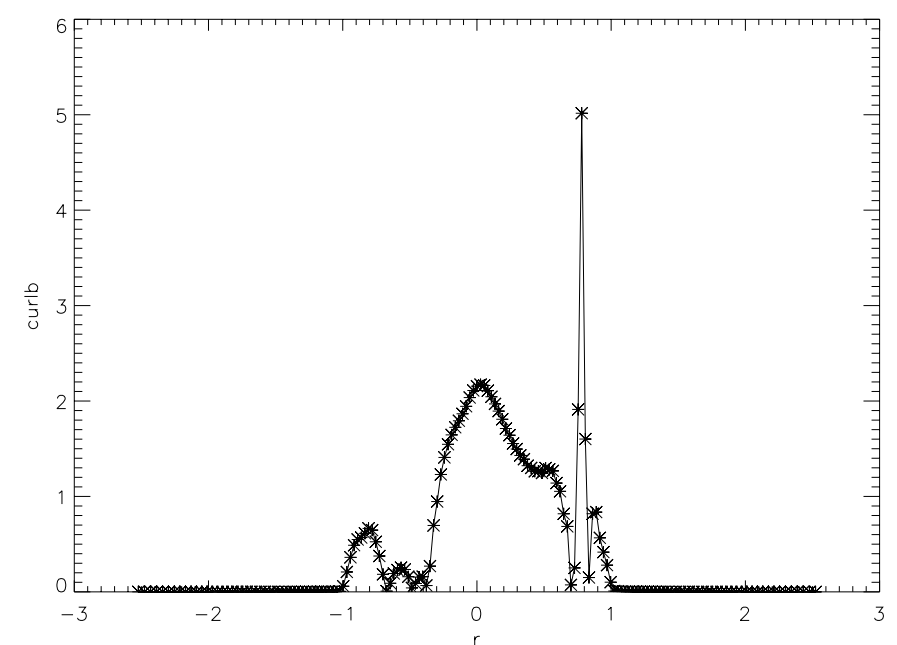

Fig. 3. Current build-up - plot of $|j|$ showing background current and a spike of current at $r=0.75$.

We also find that the growth rate of the instability, $\gamma=0.11$, is in good agreement with the value predicted by the linear results $\left(\gamma^{2}=0.01\right)$. As time increases the 
Table 1. Scaling of the maximum of the current with higher resolution.

\begin{tabular}{|c|c|c|c|c|}
\hline & \multicolumn{4}{|c|}{ grid scalings } \\
\hline \hline$n x, n y, n z$ & $81^{3}$ & $121^{3}$ & $161^{3}$ & $251^{3}$ \\
\hline$j_{\max }(\beta=0.0)$ & 8 & 11 & 15 & 29 \\
Expected scaling & 7.3 & 11 & 14.6 & 22.8 \\
\hline$j_{\max }\left(\beta=1 \times 10^{-4}\right)$ & 8.5 & 10 & 14 & 23 \\
Expected scaling & 6.7 & 10 & 13.3 & 20.7 \\
\hline$j_{\max }\left(\beta=1 \times 10^{-3}\right)$ & 8 & 10 & 13.5 & 24 \\
Expected scaling & 6.7 & 10 & 13.3 & 20.7 \\
\hline$j_{\max }\left(\beta=1 \times 10^{-2}\right)$ & 5.5 & 7.5 & 8.1 & 12.5 \\
Expected scaling & 5.0 & 7.5 & 10.0 & 15.6 \\
\hline
\end{tabular}

current build-up can be observed as a helical sheet wrapped around the kinked equilibrium current as shown in Fig. 4. Figure 5 shows a surface plot of the current at $z=0$ with the current build-up clearly much larger than the equilibrium current profile.

We have compared these results to those (Longbottom and Bennett, private communication) from the Lagrangian code. We have run these Lagrangian code simulations using Equilibrium 1 as the initial equilibrium and using the same velocity perturbation as for the simulations using Lare3d. For the $\beta=0$ case we find a maximum current of 50, 25 times the maximum equilibrium current. As can be seen from Fig. 5 the Lagrangian code simulations also show a large build up of current.

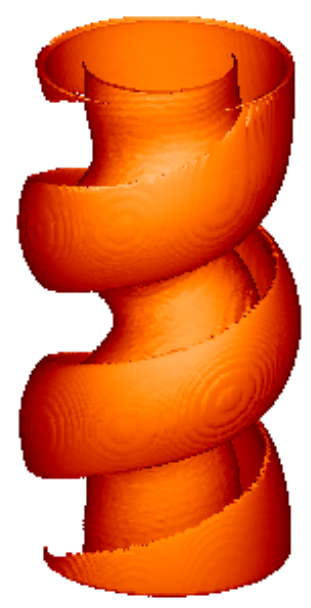

Fig. 4. Isosurface of $|j|$ from Lare3d.

For the simulations carried out using Lare3d, on the $81^{3}$ grid the maximum value of the current is found to be 8.0 whilst on the $161^{3}$ grid the maximum of the current is 15.0 agreeing well with the expected scaling which would give $\frac{161}{81} \times 8=15.9$. Whereas for $\beta=10^{-2}, j_{\max }$ is 6.0 on the $81^{3}$ grid and 7.5 on the $121^{3}$ grid. The scaling of $j_{\max }$ with higher resolution is shown more clearly, for all the values of $\beta$ considered, in Table 1 . We can see that the current does scale with increased resolution for the $\beta<$ $10^{-2}$ cases. We can therefore state that for Equilibrium 1 which has a shear of 6.32 we do not observe saturation of the current for $\beta<10^{-2}$.

\subsubsection{Resistive evolution}

To investigate the effect of resistivity on the evolution of the instability we carry out the simulations on a $121^{3}$ grid for 60 Alfvén times for the $\beta=0$ case and for 80 Alfvén times for $\beta=0.01$. The $121^{3}$ grid gives us reasonably high resolution without taking too much computational time. We do not run the simulation until the current drops back below $j_{\text {crit }}$ as has been done in other simulations (Baty 2000a). Instead the simulation is run until the total accumulated ohmic heating levels off to an (almost) constant value, i.e. resistive effects become negligible.

The resistivity has the form, given in Eq. (16) and in this case we take $j_{\text {crit }}=4.0$ since this is twice the equilibrium current value and $\eta_{0}=10^{-4}$. This keeps the anomalous resistivity, the resistivity which we have added to the code to simulate the resistivity in the plasma, small as would be expected in the solar corona but ensures that it is larger than the numerical resistivity, inherent in the numerical scheme.

With resistivity included in the code, and indeed localised at current concentrations, the code allows reconnection and diffusion of magnetic field lines. In reality this would have occurred on a physically realistic timescale provided the current concentration seen in the code is sufficiently large, i.e. for the purposes of this paper the numerical current concentration can be imagined to be a current sheet.

For $\beta=0$ the resistivity is switched on after approximately $t=25$ Alfvén times when the current exceeds the critical value. The ohmic heating then increases until $t=40$ when it settles to an almost constant value. The current has a maximum value of $j_{\max }=8.0$ at $t=40$ and then decreases, reaching a value of 6.0 at $t=60$ when we stop the simulation.

During the non-linear evolution of the instability the central column of current is shifted outwards into the current sheet region as shown in Fig. 6 at $t=30$. It is in this region that we would expect reconnection to occur. The current drops between $t=40$ and $t=55$, when the ohmic heating reaches a constant value, indicating that reconnection is taking place. Figure 7 shows a selection of magnetic fieldlines at $t=0$ and the same fieldlines at $t=50$, identified by their location on the lower boundary. It can be seen that the reconnection has resulted in the fieldlines becoming untwisted.

We calculate the amount of free magnetic energy released by considering the energy in the $B_{\theta}$ component of the magnetic field. We find that $41 \%$ of the free magnetic energy is released. 

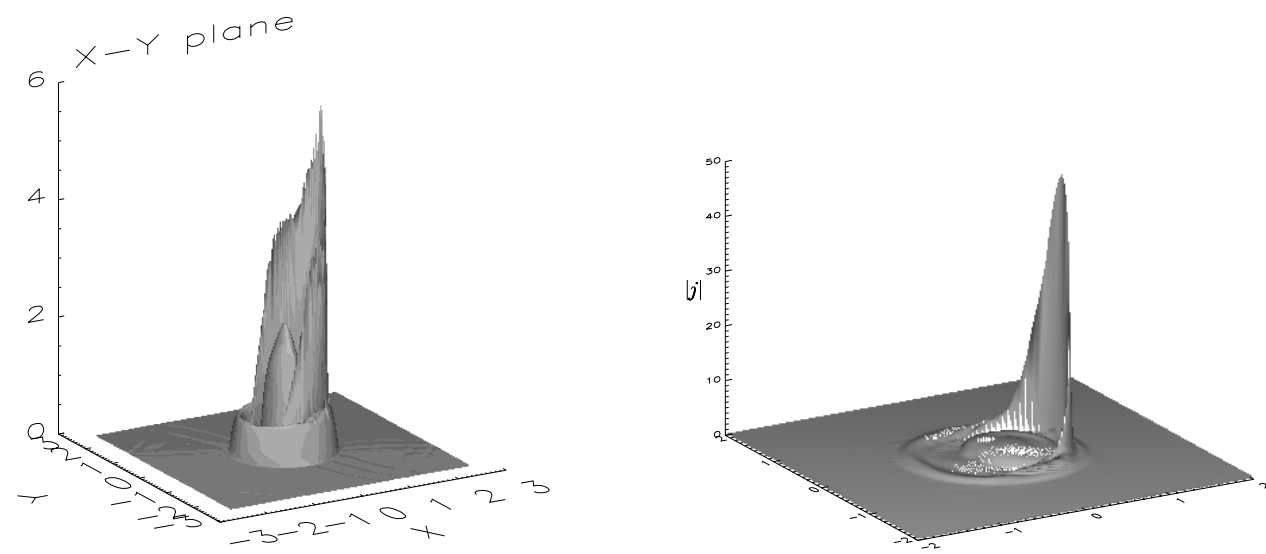

Fig. 5. Surface plots of current at $z=0$ from Lare3d (left) and the Lagrangian code (right).

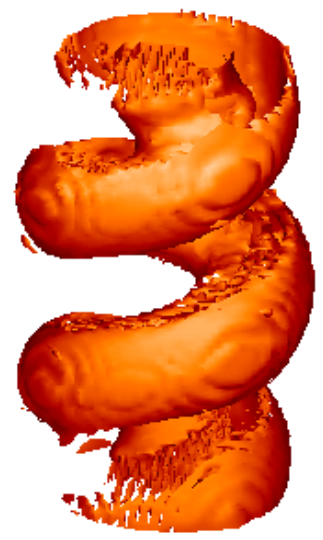

Fig. 6. Isosurface of $|j|=1.2$ at $t=30$ for the resistive evolution.

As for the ideal non-linear evolution the $\beta=0.01$ case evolves more slowly because of its lower growth rate. For this case we run the simulations for 80 Alfvén times. The central column of current is shifted outwards into the current concentration region as in the previous case but at a time of 40 Alfvén times. The current exceeds the critical value at $t=35$ Alfvén times and has a maximum of 8.0 at $t=62$. It then drops to a value of 5.6 at $t=74$. The reconnection releases $47 \%$ of the free magnetic energy. This compares favourably with the results of Arber et al. (1999) which suggested $54 \%$ and those of Baty (2000) which suggested that $57 \%$ of the free magnetic energy was released.

Therefore, we find that during the resistive evolution of the instability reconnection occurs for both $\beta=0$ and $\beta=10^{-2}$. The only effect of taking the plasma $\beta$ to be zero (a value which is clearly unphysical) is to speed up the evolution of the instability and so reduce the computational time.

\subsection{Equilibrium 2}

\subsubsection{Non-linear evolution}

We run the code using Equilibrium 2 as the initial equilibrium for 45 Alfvén times on $81^{3}, 121^{3}$, and $161^{3}$ grids. We choose different values for $a, \Phi_{0}$ and $b$ thus varying the shear of the configuration. The results are summarised in Table 2 along with the scalings which we would expect based on the $121^{3}$ grid results. The value of $j_{\max }$ is calculated by finding the maximum of $|j|$ and storing it at each timestep. The maximum of these between $t=0$ and $t=45$, where the simulations are stopped, is then taken to be $j_{\max }$. We take this maximum value rather then the value at $t=45$ because once the current has reached the maximum numerical resistivity can cause it to diffuse away.

The shears quoted are calculated at the radius where the numerical results show the current concentration forming, namely at the mode rational surface. We consider initial equilibria for which the shear varies between 1.67 and 9.42 .

This allows us to investigate the relationship between the shear of the loop and the formation of current sheets. Our results show no saturation of the current for runs 1 , 2, 3 and 5 . In fact runs 1, 2, 3 and 5 show better than expected scaling of the current with higher resolution implying current sheet formation. For example, consider run 3 with a shear of 2.02 at the mode rational surface. The maximum value of $j \mid$ is 9.8 on the $81^{3}$ grid, 14.0 on the $121^{3}$ grid and 19.0 on the $161^{3}$ grid. This compares favourably with the expected values of $9.4,14.0$, and 18.6 respectively. However, runs 4, 6, and 7 have lower values of $j_{\max }$ than was expected and, therefore, require further investigation.

We have carried out higher resolution simulations on some of these configurations. From the table it appears that runs 1-4 show no sign of current saturation whereas runs 5-7 may do so. Therefore, we take one example from runs $1-4$ and one from runs 5-7 and carry out the simulations on higher resolution. In these cases we run the simulations on a $251^{3}$ grid stretched as before. For run 7 we found $j_{\max }$ to be 24.0 . This value is exactly the same as that for the $161^{3}$ grid suggesting that the current is saturating and that we do not observe current sheet formation. However, for run 2 the maximum current is 26.0. 

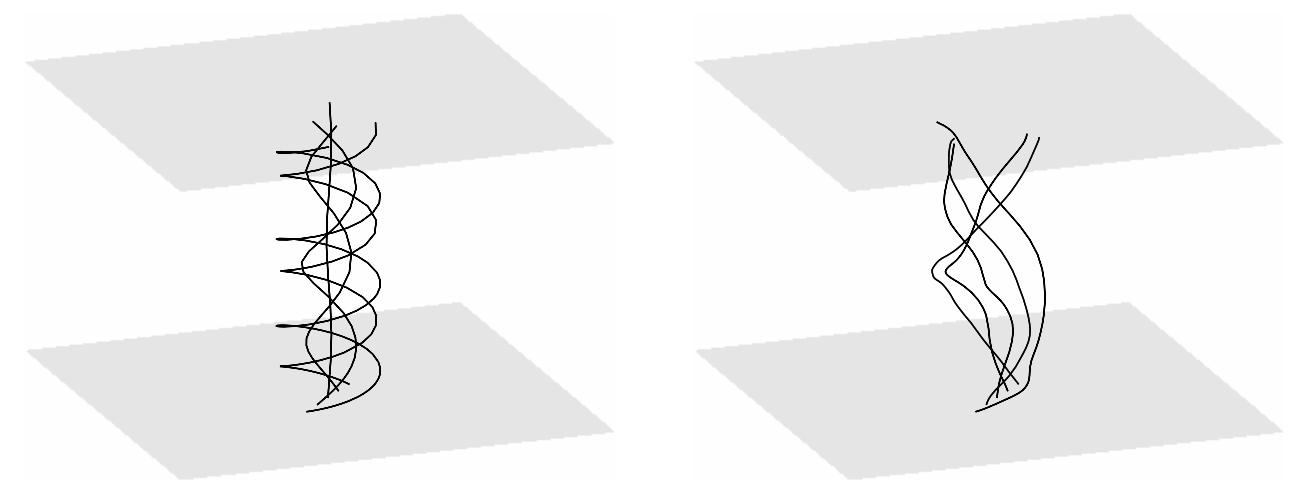

Fig. 7. A selection of fieldlines at $t=0$ (left) and at $t=50$ (right).

Table 2. Configurations investigated and their respective shear, growth rates and maximum values of the current.

\begin{tabular}{|c|c|c|c|c|c|c|c|c|}
\hline run & $a$ & $\Phi_{0}$ & $b$ & shear & $\gamma$ & $\begin{array}{c}j_{\max } \\
\left(81^{3}\right)\end{array}$ & $\begin{array}{c}j_{\max } \\
\left(121^{3}\right)\end{array}$ & $\begin{array}{c}j_{\max } \\
\left(161^{3}\right)\end{array}$ \\
\hline \hline run 1 & 0.5 & 1.0 & 2.0 & 1.67 & 0.07 & 2.3 & 3.2 & 4.5 \\
\hline expected scalings (based on $121^{3}$ results) & 2.1 & 3.2 & 4.3 \\
\hline \hline run 2 & 0.3 & 1.2 & 2.0 & 1.70 & 0.1 & 7.9 & 10.0 & 13.5 \\
\hline expected scalings (based on $121^{3}$ results) & 6.7 & 10.0 & 13.3 \\
\hline \hline run 3 & 0.0 & 1.5 & 2.0 & 2.02 & 0.11 & 9.8 & 14.0 & 19.0 \\
\hline expected scalings (based on $121^{3}$ results) & 9.4 & 14.0 & 18.6 \\
\hline \hline run 4 & 0.5 & 1.5 & 1.2 & 2.86 & 0.12 & $/$ & 17.0 & 19 \\
\hline expected scalings (based on $121^{3}$ results) & 11.4 & 17.0 & 22.6 \\
\hline \hline
\end{tabular}

This is twice the value on the $161^{3}$ grid suggesting that the current is not saturating.

For this set of equilibria the trend suggests that for higher shear the current saturates. Lower values of shear have the scaling indicative of current sheet formation. However for lower shear $j_{\max }$ is lower so caution is required here as this may saturate at higher resolution.

\subsubsection{Resistive evolution}

We carry out the resistive simulations for run 2 and run 5 with $\beta=0$.

The resistive simulations for run 5 are run until $t=60$ on a $121^{3}$ grid. This gives reasonable grid size and would be expected to allow enough time for the ohmic heating to steady off to a constant value as for Equilibrium 1. However, the ohmic heating continues to increase throughout the simulation and in fact increases after $t=50$.
The current exceeds the critical value at about $t=25$, switching on the resistivity. It then increases to a value of 13.0 at $t=40$ before decreasing to a value of 10.0 at $t=50$ and then increasing again to reach a value of 14.5 at $t=58$. Only $33 \%$ of the free magnetic energy has been released by $t=60$ when the simulation is stopped.

We run the simulation of the resistive evolution of the instability for run 2 on an $81^{3}$ grid. This allows us to run the simulation for longer, in this case for 100 Alfvén transit times. The behaviour of the current and the ohmic heating during the evolution is illustrated by Fig. 10. The resistivity is switched on at $t=30$ when the current exceeds $j_{\text {crit }}$. The ohmic heating then increases steadily until $t=60$ where it planes off. It takes a constant value until $t=80$ where it increases steeply and is still increasing at $t=100$ where the simulation is ended. The current increases to a value of 7.0 at $t=40$ and then remains at that value until $t=70$ when it starts increasing again reaching a value of 15.0 at $t=100$. In this case only $15 \%$ of the free magnetic energy is released. 


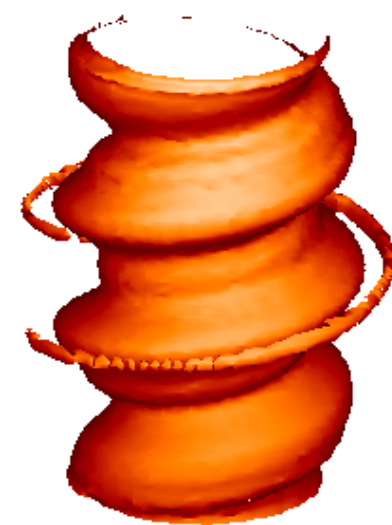

Fig. 8. The isosurface of the current for run 1 with the current concentration seen as a thin thread wrapped around the central current.

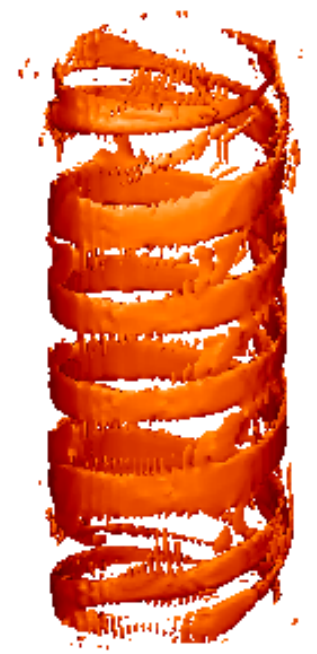

Fig. 9. The isosurface of the current for run 7 showing the current concentration as a thicker helical ribbon.

We have no clear explanation of this behaviour at present. This will be the subject of a separate investigation which we defer until a later date.

\section{Discussion and conclusions}

Our aim in this paper has been to consider the question of current sheet formation during the non-linear evolution of the $m=1$ kink instability. In particular, we have investigated the effect of shear on current sheet formation and therefore on magnetic reconnection. To do this we have carried out numerical simulations of the non-linear and resistive evolution of the kink instability for two equilibria. Equilibrium 1 has a shear of 6.32 and Equilibrium 2 allows us to vary the shear.

For Equilibrium 1 we find a scaling which is indicative of current sheet formation during the non-linear evolution of the kink instability for $\beta<10^{-2}$. We have carried out simulations on four different grid resolutions and found that the current does scale with higher resolution as would be expected for a current sheet. Furthermore the
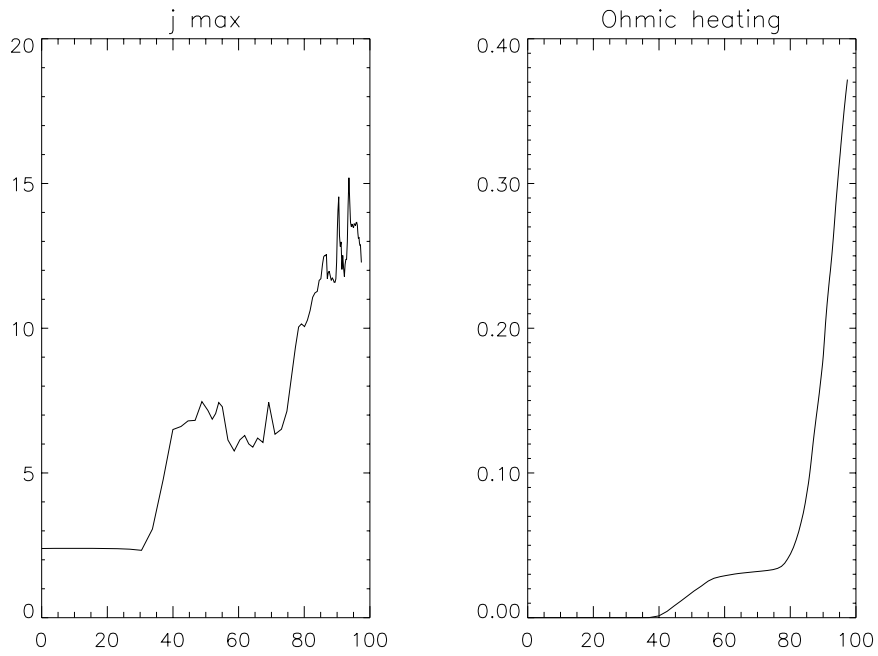

Fig. 10. Plots of the current and ohmic heating during the resistive evolution of the instability for run 2 .

results from the Lagrangian code confirm this. There is no sign of saturation of the current. During the resistive evolution reconnection takes place releasing $41 \%$ of the free magnetic energy in the $\beta=0$ case and $47 \%$ in the more physical $\beta=0.01$ case. This is in good agreement with previous results such as those of Arber et al. (1999) who found that $54 \%$ of the free energy is released.

For Equilibrium 2 the evolution appears to be more complicated. As can be seen from Table 2 runs 1, 2, 3, and 5 seem to show scaling indicative of current sheet formation whereas runs 4, 6 and 7 indicate saturation of the current. During the resistive evolution the ohmic heating steadies off but then increases again and is still increasing when the simulations are stopped. Similarly the current increases to a certain value, remains constant for some time but then increases again as shown in Fig. 10. This contrasts with Equilibrium 1 where the ohmic heating steadies off to a constant value and does not increase again and the current peaks and then falls off. For Equilibrium 1 we find that $41 \%$ of the free magnetic energy is released for the $\beta=0$ case but for Equilibrium 2 only $33 \%$ of the energy is released for run 5 and just $15 \%$ for run 2 .

Given these results it is worth considering the differences between Equilibrium 2 and Equilibrium 1, for which we did not observe current saturation and the differences between those configurations of Equilibrium 2 which do indicate current sheet formation and those for which the current saturates. Since Equilibrium 1 has a shear of 6.32 and the shear for Equilibrium 2 varies between 1.76 to 9.42 the value of the shear of the loop is unlikely to affect the formation of the current sheets. One obvious difference between Equilibrium 1 and Equilibrium 2 is in the current concentrations themselves. From Fig. 4 we can see that the current concentration developed during the non-linear evolution of the kink instability for Equilibrium 1 is an axially wide sheet wrapped around the kinked central column of current. This is similar to the current sheets found by Arber et al. (1999). In contrast the current concentrations 
observed for Equilibrium 2 are, as shown in Figs. 8 and 9 , axially thin and thread-like. Another difference is in the twist profiles. The second equilibrium is defined in such a way that the twist is constant for $r<a$ and then decreases monotonically. However this means that for $r<a$ the field is, in fact, the Gold-Hoyle field which does not have a mode rational surface and so does not form current sheets (Baty 1997b). While Equilibrium 2 is not entirely a constant twist field and does have a mode rational surface it may be that the region of constant twist does affect the formation of current sheets and therefore reconnection.

In conclusion,

- Our results show that Equilibrium 1 demonstrates the same behaviour as the equilibrium discussed in Arber et al. (1999) but it has smaller shear.

- The current does scale with higher resolution for Equilibrium 1 for $\beta<10^{-2}$ indicating current sheet formation.

- Once resistivity is included in the simulations reconnection occurs releasing nearly half of the free magnetic energy. This is sufficient energy to explain a compact loop flare.

- The results from Equilibrium 2 suggest that current sheet formation does not occur for all equilibria but does not simply depend on the shear of the loop, as was suggested by Baty (2000).

- The shape and magnitude of the current is dependent on the internal structure of the loop in a more complex manner than was previously suggested.

- If the current does not saturate for an equilibrium then reconnection will release the amount of energy required for a compact loop flare.

Acknowledgements. The authors would like to thank Aaron Longbottom and Keith Bennett for providing the Lagrangian code simulations and for many useful discussions. The authors would like to thank Gordon Petrie for providing the fieldline plotting routine and Gordon Petrie and Steve Brooks for their assistance in using it. The authors would also like to thank the anonymous referee for many useful comments. The simulations were carried out on the UK MHD consortium compaq cluster at the University of St Andrews funded by JREI/SHEFC.

\section{References}

Arber, T. D., Longbottom, A. W., Gerrard, C. L., \& Milne, A. M. 2001, J. Comput. Phys., submitted

Arber, T. D., Longbottom, A. W. \& Van der Linden, R. A. M. 1999, ApJ, 517, 990

Baty, H. 1997a, A\&A, 318, 621

Baty, H. 1997b, Solar Phys., 172, 249

Baty, H. 2000a, A\&A, 353, 1074

Baty, H. 2000b, A\&A, 360, 345

Baty, H., \& Heyvaerts, J. 1996, A\&A, 308, 935

Baty, H., Einaudi, G., Lionello, R., \& Velli, M. 1998, A\&A, 333,313

Bazdenkov, S., \& Sato, T. 1998, ApJ, 500, 966

Bychenkov, V. Yu., Silin, V. P., \& Uryupin, S. A. 1988, Phys. Rep., 164, 119

Caramana, E. J., Shashkov, M. J., \& Whalen, P. P. 1998, J. Comput. Phys., 144, 70

Evans, C. R., \& Hawley, J. F. 1988, ApJ, 332, 659

Goedbloed, J. P. 1983, Rijnhuizen Rep., 83, 145

Gold, T., \& Hoyle, F. 1960, MNRAS, 120, 89

Hood, A. W. 1986, Solar Phys., 105, 307

Lionello, R., Velli, M., Einaudi, G., \& Mikic, Z. 1998, ApJ, 494, 840

Priest, E. R., \& Forbes, T. 2000, Magnetic Reconnection: MHD theory and applications, CUP

Stone, J. M., \& Norman, M. L. 1992, ApJS, 80, 753

Stone, J. M., \& Norman, M. L. 1992, ApJS, 80, 791

Van Der Linden, R. A. M., Hood, A. W., \& Goedbloed, J. P. 1995, Solar Phys., 154, 69

Van Leer, B. 1997, J. Comput. Phys., 135, 229

Velli, M., Einaudi, G., \& Hood, A. W. 1990, ApJ, 350, 419

Wilkins, M. L. 1980, J. Comput. Phys., 36, 281 